\title{
Nurse-Perceived Barriers to Effective Communication Regarding Prognosis and Optimal End-of-Life Care for Surgical ICU Patients: A Qualitative Exploration
}

\author{
Rebecca A. Aslakson, M.D., M.Sci., Rhonda Wyskiel, R.N., ${ }^{1}$ Imani Thornton, M.D.,, Christina Copley, R.N., ${ }^{2}$ \\ Dauryne Shaffer, R.N., ${ }^{2}$ Marylou Zyra, R.N., ${ }^{2}$ Judith Nelson, M.D., J.D., ${ }^{3}$ and Peter J. Pronovost, M.D., Ph.D. ${ }^{1}$
}

\begin{abstract}
Background: Integration of palliative care for intensive care unit (ICU) patients is important but often challenging, especially in surgical ICUs (SICUs), in part because many surgeons equate palliative care with terminal care and failure of restorative care. SICU nurses, who are key front-line clinicians, can provide insights into barriers for delivery of optimal palliative care in their setting.

Methods: We developed a focus group guide to identify barriers to two key components of palliative careoptimal communication regarding prognosis and optimal end-of-life care-and used the tool to conduct focus groups of nurses providing bedside care in three SICUs at a tertiary care, academic, inner city hospital. Using content analysis technique, responses were organized into thematic domains that were validated by independent observers and a subset of participating nurses.

Results: Four focus groups included a total of 32 SICU nurses. They identified 34 barriers to optimal communication regarding prognosis, which were summarized into four domains: logistics, clinician discomfort with discussing prognosis, inadequate skill and training, and fear of conflict. For optimal end-of-life care, the groups identified 24 barriers in four domains: logistics, inability to acknowledge an end-of-life situation, inadequate skill and training, and cultural differences relating to end-of-life care.

Conclusions: Nurses providing bedside care in SICUs identify barriers in several domains that may impede optimal discussions of prognoses and end-of-life care for patients with surgical critical illness. Consideration of these perceived barriers and the underlying SICU culture is relevant for designing interventions to improve palliative care in this setting.
\end{abstract}

\section{Introduction}

$\mathrm{O}$ VER $20 \%$ of U.S. deaths occur either in or shortly after admission to an intensive care unit (ICU) ${ }^{1}$ and multiple researchers, professional societies, and expert panels advocate for improved palliative care for all ICU patients, regardless of prognosis. ${ }^{2-4}$ The public and policy makers emphasize end-of-life care as a key area for improving overall heath care quality. ${ }^{5}$ Consequently, interventions using both integrative and consultative models ${ }^{6}$ to improve delivery of palliative care to ICU patients have been implemented and evaluated. ${ }^{7-16}$ However, few of these interventions have been tested specifically in surgical ICUs (SICUs).
For a variety of reasons, patients in SICUs are at risk for receiving inadequate palliative care. ${ }^{17-21}$ The time frame of surgical illness is often compressed, which requires patients, families, and clinicians to make difficult decisions in haste, without either clear information on patient prognosis or adequate emotional adjustment. ${ }^{21}$ SICUs are more likely to use "open" or "semiclosed" administrative models ${ }^{18}$ in which coordination of multiple care teams can be difficult. Surgical culture, with an overemphasis on clinician accountability for patient outcomes, ${ }^{22}$ a "rescue credo" amongst surgeons, ${ }^{23}$ and pride in technical, as opposed to psychosocial or spiritual aspects of patient $\mathrm{care}^{24}$ may also hamper incorporation of palliative care principles. Besides these SICU-specific issues,

\footnotetext{
${ }^{1}$ Department of Anesthesiology and Critical Care Medicine, The Johns Hopkins School of Medicine, Baltimore, Maryland.

${ }^{2}$ Department of Surgical Nursing, The Johns Hopkins Hospital, Baltimore, Maryland.

${ }^{3}$ Department of Medicine, Division of Pulmonary, Critical Care and Sleep Medicine and Hertzberg Palliative Care Institute, Mount Sinai School of Medicine, New York, New York.

Accepted March 27, 2012.
} 
fewer than $10 \%$ of all ICU patients participate in decision making; ${ }^{25}$ vulnerable surrogates must thus make emotionally laden decisions, often without adequate information about the patient's prior wishes and goals of care.

Previous studies have indicated that ICU nurses have a unique role in the dynamic between surgeons, ICU physicians, and families of critically ill patients. As compared to SICU physicians and surgeons, the nurse spends more time communicating with the patients' family regarding progno$\operatorname{sis}^{26,27}$ and often acts as "translator" $\mathrm{er}^{\prime \prime 27}$ for patients and patient families. If there is conflict between physicians and families or between varying physician groups (such as between surgeons and ICU intensivists), the nurse may serve as a "culture broker"28 attempting to mitigate conflicts. In end-of-life care, nurses help families "reconnect" with the dying patient to ensure optimal communication and a "peaceful death." 29 Yet, compared to medical ICU nurses, SICU nurses report lower levels of physician-nurse collaboration. ${ }^{30}$ They also report fewer opportunities to speak to surgeons about patient prognosis, and often feel their comments to both surgeons and intensivists are undervalued. ${ }^{17,26}$

A consensus group sponsored by the Robert Wood Johnson Foundation $^{3}$ identified seven domains for high-quality endof-life care in the ICU: patient- and family-centered decision making, communication, continuity of care, emotional and practical support of patients and families, symptom management and comfort care, spiritual support, and emotional and organizational support for ICU clinicians. Based on our experiences as SICU physicians and nurses, we believed the specific domain of "communication" and the general approach to end-of-life care were especially important in our SICU setting. We undertook this qualitative study to explore the barriers to optimal communication and end-of-life care in SICUs as perceived by the bedside nurses.

\section{Methods}

In September 2009 we convened four focus group sessions with a goal of discussing barriers to delivery of core elements of palliative care to SICU patients. We interviewed groups of SICU nurses at the Johns Hopkins Hospital who practice in one of three SICUs: a 15-bed cardiac surgical ICU that predominantly admits patients after cardiac surgery; a 13-bed surgical ICU and intermediate care unit (IMC) that predominantly admits patients after trauma, transplant, and vascular surgeries; and a 16-bed general surgical ICU and IMC that predominantly admits patients after thoracic, general abdominal, plastic, gynecologic, and ear/nose/throat surgeries. Each surgical ICU operates under a "mandatory consult" plan; patients are admitted by the primary surgeon with his or her corresponding house staff team, and with a required ICU team consultation. ${ }^{31}$ Physician medical decisions are made jointly between the primary surgical and ICU teams. The ICU attending team is interdisciplinary and comprised predominantly of physicians with primary boarding in either surgery or anesthesia and subspecialty certification in critical care. The ICU team is comprised of house staff and of nurse practitioners with responsibilities similar to those of senior house officers.

At the time of this study, the hospital's interdisciplinary palliative care team consulted only on medical-oncologic and/or medicine patients, not on surgical patients. Questions related to hospice care for a surgical patient were referred to a social worker.

\section{Item generation and refinement for focus group guide}

Our study focused on nurses' perceptions of communication regarding prognosis, specifically, and end-of-life care, more generally. Based on a review of the literature and experience, the study investigators, composed of clinicians in surgical nursing and surgical critical care, generated a list of open-ended questions; the goal of the questions was to facilitate unbiased and unrestricted discussion about these two topics across multiple focus groups. Through successive iterations, the research team reduced and refined this list to include four key questions for each topic (Table 1). For these focus groups, "prognosis" was specifically defined as how a patient's illness and overall health are likely to evolve during the hospitalization or over the next few days to months. Consequently, "prognosis" incorporated both whether or not a patient was likely to die during the hospitalization and what would be the quality of life during the hospitalization and after discharge.

\section{Focus groups}

Nurses were recruited to the focus groups through email messages to full-time nurses employed in any of the three surgical ICUs. We also posted fliers throughout the participating SICUs. SICU nurse participation in the focus groups was voluntary. Meetings were held in the conference room within each ICU.

For each focus group, a moderator (RA) encouraged discussion based on the open-ended questions in the focus group

\section{Table 1. ICU Nurse Focus Group Questions}

In this context I am specifically defining prognosis as how a patient's illness and overall health is likely to evolve during this hospitalization and/or over the next few days to months.

Communication of prognosis

a) Who talks to patients and patient families about prognosis? How do they do it? Good points? Bad points?

b) What are the barriers to effective communication about patient prognosis in your ICU?

c) Would you like to see a change(s) in the way your unit handles communication about patient prognosis? How?

d) In your ICU, who do you think should be involved in discussions about patient prognosis? Practically, who do you think can be involved?

End-of-life care

a) Tell me about end-of-life and/or palliative care for patients in your unit. How do you think it is managed? Good points? Bad points?

b) What are the barriers to effective end-of-life care in your ICU?

c) What would you like to see change in the way your unit handles end-of-life care?

d) Who do you think should be involved in end-of-life care on your unit? Practically, who do you think can be involved? 
guide. During the planning phase of the project a large number of SICU nurses expressed reluctance to speak about their views in an audio-recorded session, but nursing staff were willing to participate in sessions led by a specific moderator known to them (RA, who had rotated through each of the surgical ICUs during fellowship training and was transitioning to a faculty position), with note-taking by the moderator and an independent observer (RW, IT, or MZ). Both the moderator and the observer took extensive written notes, including exact key words and phrases used by the participants. Each focus group session lasted an hour and the topic of communication regarding prognosis was discussed first, followed by the discussion about end-of-life care.

\section{Qualitative analysis}

The written notes were de-identified, compared, and pooled. Investigators (RA, PP) used content analysis technique to identify major themes emerging in the discussions. Individual barriers articulated by participating nurses were grouped in domains reflecting these themes. Multiple members of the research team (RA, RW, MZ, IT) met to reach consensus on interpretation of the nurses' responses and on the classification scheme. Ultimately, complete agreement was reached on all domains and classifications of barriers. After internal validation of the domains by the study investigators, these domains were disseminated to a subset of 10 nurses who participated in the focus groups for verification that the domains accurately represented the actual focus group discussions.

\section{Results}

We conducted four focus groups (two in the 13-bed general surgical ICU/IMC, one in the 15-bed cardiac surgical ICU, and one in the 16-bed general surgical ICU/IMC) involving 32 nurses. This sample represented approximately $20 \%$ of nurses working in the SICUs. No nurse participated in more than one focus group. Group size ranged from 5-10 nurses per meeting. In general, our cohort consisted of experienced ICU nurses, with a median of 8 (range, 0.5-30) years of ICU experience. Nearly all nurses attending the focus group sessions contributed at least one comment during the meeting.

Nurses in each unit had serious concerns about both the quality and the quantity of communication regarding prognosis and the end-of-life care provided in each respective ICU. They identified four domains of barriers to optimal communication about prognosis (Table 2):

- Logistics; physical and/or temporal impediments - "The patient is often intubated and unable to participate in the conversation;" "Surgeons have cases during the day and are not available when families are present;" "The attending is in a hurry [and]...frequently interrupted."

- Discomfort with discussing prognosis; health care providers and/or families being uncomfortable with discussions regarding prognosis - "Some people fear legal ramifications of bad outcomes and do not want to discuss prognosis;" "The goals of care were unclear even before the surgery - for both the surgeon and the patient;" "Updates from the primary [surgeon] are often unrealistic and only portray the 'small victories' instead of the overall prognosis;" "No time to talk to patients on rounds surgeons are rushed;" "Lack of formal family meetings."
TABLE 2. BARRIERS TO COMMUNICATION Regarding Prognosis

Logistics

Surgical team rounds before the family is present

Cannot assemble entire team (ICU doctors, surgeons, nurses)

Not all parties (ICU doctors, surgeons, nurses) present when meetings do occur

Other support resources not always available (social work, pastoral care, palliative care)

Not enough time during meeting

Poor availability of doctors or family for a meeting

Multiple decision makers in a family

Surrogate decision maker not at the meeting

Meetings interrupted by health care provider pagers and/or cell phone calls

Lack of unbiased person

Patient cannot participate in conversations

Unclear what prior specialists and consultants have said regarding prognosis

Discomfort with discussion

Physician discussions with nurses and families are inconclusive

Family members do not want to "hear bad news" and avoid the meeting

Prognoses are unrealistic and often portray "small victories" instead of overall prognosis

Unclear whose role it is to discuss prognosis and no one ends up doing so

Poorly defined goals of care, even prior to surgery - for surgeon and patient

Perceived lack of skill or training

Physician discussions are rushed

Families are not given adequate time to ask questions

Communication is done "last minute," often before a procedure

Families are unaware of a patient's diagnosis

There is no accepted protocol about when and what to communicate

If families do not ask for meetings, they will not receive them Physicians both use language that the family do not understand and do not recognize it

Families do not remember to ask all their questions

Families do not know what resources are available to them Fear of legal ramifications of bad outcomes

Fear of conflict

Different opinions about prognosis between care providers Inconsistencies between team members in communicating prognosis to families

Surgery and ICU teams rarely discuss prognosis but get angry when nurses discuss it

Difficult personalities of some health care providers

- Inadequate skill and training; care providers not being aware of how best to conduct discussions regarding prognosis, care providers having misconceptions about the consequences of these discussions, and/or family members not being prepared for the discussions - "Patient and patient families have difficulty forming their questions and asking about their concerns;" "The doctor does not realize when a family 
does not understand what is being said;" "Poor bedside manner by surgeons."

- Fear of conflict; care providers and/or family members avoiding conversations due to anticipated variations in prognosis that could lead to conflict amongst involved parties - "You don't know how honest a surgeon has been previously in talking with a family, especially if a patient is 'offpathway;" "Nurses don't know what was discussed with the patient preop and what their expectations are;" "ICU team is sometimes not invited to participate in the family meeting;" "Everyone is not on the same page."

The nurses also identified four domains of barriers to optimal end-of-life care (Table 3):

- Logistics; temporal factors, such as the short time when endof-life care might be provided, as well as practical factors,

TAble 3. Barriers to Optimal End-of-Life CARE IN THE SICU

\section{Logistics}

Often only a small window when can offer end-of-life care

Prognosis is often uncertain

Patient often cannot be involved in discussions

Lack of palliative care service for surgical patients

Unclear patient advance directives

Unclear surrogate decision maker for patient

Few other care resources beyond an ICU for patients on advanced life support

Inability to acknowledge an end-of-life situation

Families are often given "false hope"

Care providers often have "false hope"

Differences in opinions amongst care providers regarding prognosis

\section{Education}

Poor communication between care providers

Care providers uncomfortable discussing end-of-life care

Poor knowledge concerning available resources (chaplaincy, palliative care, hospice)

Not all care options are understood and/or presented

Poor care provider training in providing end-of-life care

Families do not understand the technologies used in ICU to keep patient "alive"

Cultural barriers

Care providers and families uncomfortable discussing end-of-life care

Prognosis is often uncertain-patient death is seen as a defeat by care providers and/or families

Hospice is rarely discussed as a care option

End-of-life situations are particularly difficult when the patient is younger

Disagreements about whether the patient should be involved in the discussion

Patient cultural beliefs that contrast with those of the care providers

Unclear as to what is a "natural death" or what are "extreme measures" or "futile

Measures," particularly when a patient is already on life support in an ICU such as the lack of a palliative care consultation service for surgery patients - "No palliative care service for surgical patients"; "No standardized order set for end-of-life care;" "Current drug infusion limits, such as max doses for pressor doses, are already not followed;" "There are no other care resources for patients beyond this ICU, especially for VAD [ventricular assist device] patients."

- Inability to acknowledge an end-of-life situation; care providers not acknowledging that the patient might die - "Doctors have different standards for what end-of-life care means;"; "Physicians are slow to acknowledge that it is end-of-life care;" "False hope given;" "It is unclear as to what is a 'natural death', especially in this ICU with patients on VAD."

- Inadequate skill and training; care providers not aware of how best to provide end-of-life care - "Nurses don't know what resources are available;" "No [nurse] training in end-oflife care;" "[Doctors] prolong [end-of-life care] and miss the window to address leaving with dignity;" "[Poor] doctor training in communication;" "Nurses don't know what to say to a family after [a patient has been] told that [a tumor] is unresectable;" "Don't know what the next steps are after the ICU;" "What is hospice?" "Families do not understand the implications of technologies used in the ICU."

- Different cultures concerning end-of-life care; care providers and/or family members not feeling comfortable with end-oflife care due to the culture of the patient and patient family and/or the culture of the SICU - "Disagreements exist about whether the patient should be involved in the discussion;" "Family choosing not to be honest with patient about diagnosis;" "Unclear what constitutes 'extreme measures' or 'futile measures,' especially when a patient already [is] with a VAD;" "Some of what we already do and do successfully is on the barrier of what is possible;" "Differences in what patients, patient families, and health care providers consider to be good 'quality of life.'"

Some other quotations highlight key nurse concerns and illustrate their discomfort with discussing these issues. "You're cornered as a nurse...it's not the nurse's place to prognose [sic] but to give possibilities" and still "a lot of conversations get dumped on the nurse." "Talking about prognosis is not always well received by the surgical attendings, PAs (physician assistants), or NPs (nurse practitioners)." Patients are "not told the truth about being in an end-of-life scenario" and "We have the sickest patients in this unit."

\section{Discussion}

In this qualitative study, bedside nurses from three SICUs identified barriers for delivery of high-quality palliative care, with barriers addressing key domains such as inadequate education or skill in palliative care, logistical difficulties, and patient and SICU cultural differences and difficulties discussing end-of-life care. Some barriers are unique to ICUs, such as "few other care resources beyond an ICU for patients on advanced life support;" other barriers are more universal for dying patients, such as "unclear patient advance directives." Many barriers applied to both communication regarding prognosis and optimal end-of-life care. This crossover could be due to conversations being convened with the 
same groups of nurses at the same time. However, it also could be because, in SICU culture, communication regarding prognosis implies potential end-of-life discussions and decisions.

Technical or logistical concerns pose significant barriers to palliative care in SICUs. Because most SICU patients cannot communicate, clinicians may have difficulty understanding their goals of care, obtaining advanced directives, and identifying surrogate decision makers. Improved preoperative "advance care planning," such as preoperative discussions to clarify patient goals, fears, and choice of surrogate, could possibly circumvent some of these barriers. Other technical barriers could be mitigated by clarifying which SICU patients require a formal family meeting, when this meeting should occur, and who should attend.

Even after technical barriers are addressed, multiple educational barriers remain. Nurses noted that they lacked training in communication regarding prognosis and optimal end-of-life care and that they thought that physicians also lacked these skills. Indeed, the nurses thought that communication by physicians, particularly in the form of family meetings and end-of-life care was often done quickly, inadequately, and ineffectively. Moreover, nurses believed that families were not being educated in what to expect for communication and how to communicate effectively with health care providers. These are multiple potential targets for future interventions.

The barriers to palliative care offered by the SICU nurses are consistent with previous observations about SICU and surgical culture as well as consistent with our hypotheses about why palliative care in a surgical culture is often challenging. In providing palliative care, each group of key SICU clinicians - the surgeon, the SICU intensivist, and the SICU nurse - is hampered for different reasons. "Total care" or "ownership" of the surgical patient, including control over key decisions, has been documented as an integral part of surgical culture. ${ }^{19,22}$ This "total care" yields great benefit to many surgical patients and has, without a doubt, advanced the field of surgery in numerous ways. However, with palliative care and specifically with end-of-life care, such "total care" can be counterproductive, particularly if the participating surgeon sees a transition to end-of-life care as a "failure." 22 Furthermore, optimal palliative care requires multiple, frequent, unhurried conversations about goals-ofcare, symptom-management, and prognosis; many surgeons, who already balance time-intensive operating room, clinic, and inpatient rounding schedules, do not have the time for these meetings. ICU physicians are hampered in that they frequently rotate on and off service; even assuming a semiopen or closed administrative model, ${ }^{18}$ they frequently direct a patient's care for only a few days to a week - an often inadequate duration of time to build sufficient rapport with a patient and family. SICU nurses could direct palliative care discussions; however, previous studies note that patients want prognostic information from physicians ${ }^{32}$ and even cite physicians to have an obligation to convey this information. ${ }^{33}$ Moreover, each of the above scenarios presumes that the surgeon, intensivist, and/or SICU nurse have the desire and skill set to adequately conduct palliative care related meetingsa presumption that some experts question. ${ }^{20,22}$

There are multiple limitations to this study. First, we studied staff from surgical ICUs in one academic medical center; our findings may not be representative of the views of nurses working in other settings. Second, it is possible that the views of our participating SICU nurses did not represent those of others who did not participate in the focus groups. Third, although we provided a working definition of "prognosis" for purposes of the discussion, clinicians may have understood the term differently based on their prior experience. Fourth, perhaps some of the identified barriers would not be valid if there was a palliative care team available to consult on SICU patients. Fifth, the participation of an investigator/clinician as moderator could have influenced both which nurses participated in the study and what they said during the focus groups; this potential problem was unavoidable given the nurses' selection of the investigator as their favored moderator. Finally, because we used written note taking rather than audio recording with verbatim transcription, we could not accurately count the frequency with which specific themes were identified. Like all qualitative work, however, this study is hypothesis-generating. Future studies including projects involving larger cohorts and quantitative methods must be designed and evaluated to further test hypotheses suggested by this work.

Nurses providing bedside care identified several specific barriers to delivering optimal palliative care to SICU patients. To overcome technical barriers, changes to policies, procedures, staffing, or training may be effective. Other barriers, such as belief in the need for the surgeon to provide "total care" to the patient or that palliative care implies "failure," relate to the culture of the SICU and will likely need further discussion. Practitioners and policy makers seeking to improve palliative care should address these barriers in design and implementation of interventions.

This study suggests multiple potential intervention targets for improving palliative care in the SICU. Such culture change requires both transparency concerning rationale and goals as well as closely coordinated teamwork between clinician groups. All potentially affected clinicians - nurses, surgeons, intensivists, palliative care specialists - are passionate patient advocates who individualize care and desire the best for their patients. By working together they may better ensure that patient goals are met, particularly regarding provision of optimal palliative care in the SICU.

\section{Acknowledgments}

Dr. Aslakson's salary was supported by a T32 National Institutes of Health grant throughout this study. The Johns Hopkins University School of Medicine institutional review board (IRB) approved this research.

\section{Author Disclosure Statement}

No competing financial interests exist.

\section{References}

1. Angus DC, Barnato AE, Linde-Zwirble WT, Weissfeld LA, Watson RS, Rickert T, Rubenfeld GD; Robert Wood Johnson Foundation ICU End-Of-Life Peer Group: Use of intensive care at the end of life in the united states: An epidemiologic study. Crit Care Med 2004;32:638-643.

2. Lanken PN, Terry PB, Delisser HM, Fahy BF, HansenFlaschen J, Heffner JE, Levy M, Mularski RA, Osborne ML, 
Prendergast TJ, Rocker G, Sibbald WJ, Wilfond B, Yankaskas JR; ATS End-of-Life Care Task Force: An official American Thoracic Society clinical policy statement: Palliative care for patients with respiratory diseases and critical illnesses. Am J Respir Crit Care Med 2008;177:912-927.

3. Mularski RA, Curtis JR, Billings JA, Burt R, Byock I, Fuhrman C, Mosenthal AC, Medina J, Ray DE, Rubenfeld GD, Schneiderman LJ, Treece PD, Truog RD, Levy MM: Proposed quality measures for palliative care in the critically ill: A consensus from the Robert Wood Johnson Foundation critical care workgroup. Crit Care Med 2006;34:S404-411.

4. Truog RD, Campbell ML, Curtis JR, Haas CE, Luce JM, Rubenfeld GD, Rushton CH, Kaufman DC; American Academy of Critical Care Medicine: Recommendations for end-of-life care in the intensive care unit: A consensus statement by the American College [corrected] of critical care medicine. Crit Care Med 2008;36:953-963.

5. Priority Areans for National Action: Transforming Health Care Quality. Adams K, Corrigan JM (eds): Washington, DC: National Academy Press, 2003.

6. Nelson JE, Bassett R, Boss RD, Brasel KJ, Campbell ML, Cortez TB, Curtis JR, Lustbader DR, Mulkerin C, Puntillo KA, Ray DE, Weissman DE; Improve Palliative Care in the Intensive Care Unit Project: Models for structuring a clinical initiative to enhance palliative care in the intensive care unit: A report from the IPAL-ICU project (improving palliative care in the ICU). Crit Care Med 2010;38:1765-1772.

7. Lilly CM, De Meo DL, Sonna LA, Haley KJ, Massaro AF, Wallace RF, Cody S: An intensive communication intervention for the critically ill. Am J Med 2000;109:469-475.

8. Lilly CM, Sonna LA, Haley KJ, Massaro AF: Intensive communication: Four-year follow-up from a clinical practice study. Crit Care Med 2003;31:S394-399.

9. Lautrette A, Darmon M, Megarbane B, Joly LM, Chevret S, Adrie C, Barnoud D, Bleichner G, Bruel C, Choukroun G, Curtis JR, Fieux F, Galliot R, Garrouste-Orgeas M, Georges H, Goldgran-Toledano D, Jourdain M, Loubert G, Reignier J, Saidi F, Souweine B, Vincent F, Barnes NK, Pochard F, Schlemmer B, Azoulay E: A communication strategy and brochure for relatives of patients dying in the ICU. NEJM 2007;356:469-478.

10. Campbell ML, Guzman JA: Impact of a proactive approach to improve end-of-life care in a medical ICU. Chest 2003; 123:266-271.

11. Campbell ML, Guzman JA: A proactive approach to improve end-of-life care in a medical intensive care unit for patients with terminal dementia. Crit Care Med 2004;32:1839-1843.

12. Curtis JR, Treece PD, Nielsen EL, Downey L, Shannon SE, Braungardt T, Owens D, Steinberg KP, Engelberg RA: Integrating palliative and critical care: Evaluation of a qualityimprovement intervention. Am J Respir Crit Care Med 2008;178:269-275.

13. Mosenthal AC, Murphy PA, Barker LK, Lavery R, Retano A, Livingston DH: Changing the culture around end-of-life care in the trauma intensive care unit. J Trauma 2008;64:1587-1593.

14. Ahrens T, Yancey V, Kollef M: Improving family communications at the end of life: Implications for length of stay in the intensive care unit and resource use. Amer J Crit Care 2003;12:317-323.

15. Norton SA, Hogan LA, Holloway RG, Temkin-Greener H, Buckley MJ, Quill TE: Proactive palliative care in the medical intensive care unit: Effects on length of stay for selected high-risk patients. Crit Care Med 2007;35:1530-1535.

16. McCormick AJ, Curtis JR, Stowell-Weiss P, Toms C, Engelberg R. Improving social work in intensive care unit pallia- tive care: Results of a quality improvement intervention. J Palliat Med 2010;13:297-304.

17. Cassell J. Life and Death in Intensive Care. Philadelphia: Temple University Press, 2005.

18. Cassell J, Buchman TG, Streat S, Stewart RM: Surgeons, intensivists, and the covenant of care: Administrative models and values affecting care at the end of life - updated...including commentary by Buchman TG and Stewart RM. Crit Care Med 2003;31:1551-1559.

19. Penkoske PA, Buchman TG: The relationship between the surgeon and the intensivist in the surgical intensive care unit. Surg Clin North Am 2006;86:1351-1357.

20. Dunn GP, Milch RA, Mosenthal AC, Lee KF, Easson AM, Huffman JL: Palliative care by the surgeon: How to do it. J Am Coll Surg 2002;194:509-537.

21. Mosenthal AC: Palliative care in the surgical ICU. Surg Clin North Am 2005;85:303-313.

22. Buchman TG, Cassell J, Ray SE, Wax ML: Who should manage the dying patient?: Rescue, shame, and the surgical ICU dilemma. J Am Coll Surg 2002;194:665-673.

23. Nuland S. How We Die. New York: Knopf, 1993, pp. 250-253.

24. Krizek T: Spiritual dimensions of surgical palliative care. Surg Oncol Clin N Am 2001;10:51.

25. Prendergast $\mathrm{T}$, Luce J: Increasing incidence of withholding and withdrawal of life support from the critically ill. Am J Respir Crit Care Med 1997;155:15-20.

26. Aslakson RA, Wyskiel R, Shaeffer D, Zyra M, Ahuja N, Nelson JE, Pronovost PJ: Surgical intensive care unit clinician estimates of the adequacy of communication regarding patient prognosis. Crit Care 2010;14:R218.

27. Asch DA, Shea JA, Jedrziewski MK, Bosk CL: The limits of suffering: Critical care nurses' views of hospital care at the end of life. Soc Sci Med 1997;45:1661-1668.

28. Jezewski MA, Scherer Y, Miller C, Battista E: Consenting to DNR: Critical care nurses' interactions with patients and family members. Am J Crit Care 1993;2:302-309.

29. Simpson $\mathrm{SH}$ : Reconnecting: The experiences of nurses caring for hopelessly ill patients in intensive care. Intensive Crit Care Nurs 1997;13:189-197.

30. Baggs JG, Schmitt MH, Mushlin AI, Eldredge DH, Oakes D, Hutson AD: Nurse-physician collaboration and satisfaction with the decision-making process in three critical care units. Am J Crit Care 1997;6:393-399.

31. Pronovost PJ, Angus DC, Dorman T, Robinson KA, Dremsizov TT, Young TL: Physician staffing patterns and clinical outcomes in critically ill patients: A systematic review. JAMA 2002;6:2151-2162.

32. Evans LR, Boyd EA, Malvar G, Apatira L, Luce JM, Lo B, White DB: Surrogate decision-makers' perspectives on discussing prognosis in the face of uncertainty. Am J Respir Crit Care Med 2009;179:48-53.

33. Apatira L, Boyd EA, Malvar G, Evans LR, Luce JM, Lo B, White DB: Hope, truth, and preparing for death. Ann Int Med 2008;149:861-868.

Address correspondence to: Rebecca A. Aslakson, M.D., M.Sci.

The Johns Hopkins Hospital

Department of Anesthesiology and Critical Care Medicine 600 North Wolfe Street, Meyer 297A

Baltimore, MD 21287

E-mail: raslaks1@jhmi.edu 